\title{
Better late than never, but never late is better: Risk assessment of nuclear power construction projects
}

\author{
J. Portugal-Pereira ${ }^{a, b, *}$, P. Ferreira ${ }^{c}$, J. Cunha $^{c}$, A. Szklo ${ }^{a}$, R. Schaeffer ${ }^{a}$, M. Araújo ${ }^{c}$ \\ ${ }^{a}$ Energy Planning Program, Graduate School of Engineering, Universidade Federal do Rio de Janeiro, Centro de Tecnologia, Bloco C, Sala 211, Cidade Universitária, Itha \\ do Fundão, 21941-972 Rio de Janeiro, RJ, Brazil \\ ${ }^{\mathrm{b}}$ Centre of Environmental Policy, Imperial College London, Weeks Building 16-18 Prince's Gardens, South Kensington, London SW7 1BA, UK \\ ${ }^{\mathrm{c}}$ ALGORITMI Centre, School of Engineering, University of Minho, Campus Azurém, 4800-058 Guimarães, Portugal
}

\section{A R T I C L E I N F O}

\section{Keywords:}

Nuclear energy

Overnight construction costs

Financial costs

Risk assessment

\begin{abstract}
A B S T R A C T
This work seeks to evaluate overnight construction costs (OCC) and lead-time escalation of nuclear power construction projects from 1955 to 2016. To this end, a comprehensive database of commercial Light Water Reactors (LWR) was developed and a statistical analysis was conducted. Findings reveal a significant delay in lead-time, especially for the last generation reactors constructed from 2010s, with $3 / 4$ of the sample showing significant construction delays. This results in an escalation of capital costs rather than in a decline. Average OCC of newer reactors is $60 \%$ higher than the ones implemented in the earlier stages of the nuclear era. This suggests a discontinuity of the learning curve for both OCC and lead-time, which threats the market and financial sustainability of current and future nuclear energy projects. Although this is a general trend, this discontinuity is country specific and, thus, induced by national policies and regulatory frameworks. Therefore, the role of nuclear technology as an alternative to cope with the need for a decarbonisation of the power sector must be better evaluated, taking into account the real cost impacts of nuclear technology implementation.
\end{abstract}

\section{Introduction}

The glorious times of nuclear power are under pressure. In the early 1960 s and especially after the 1973 oil embargo, the nuclear power installed capacity increased steadily. The historic peak was reached in 1984-85, with the grid connection of 51 new commercial operating reactors (IAEA, 2017a). However, since the 1990s, the pace of new constructions has stagnated given low oil prices and safety concerns after the nuclear accidents in Three Mile Island (1979) and Chernobyl (1986). Recently, in the aftermath of the Fukushima Dai-ichi accident (2011), several countries have revised their nuclear policies and reinforced new regulatory requirements to strengthen safety systems of operating reactors (Aoki and Rothwell, 2013; Huenteler et al., 2012; NRA, 2013; Portugal Pereira et al., 2014; Soda et al., 2017; Srinivasan and Rethinaraj, 2013; Vivoda, 2012). Following this tendency, developed countries, traditionally forefront protagonists of nuclear energy, have phased down investments for new nuclear reactors. Confirming the recent year's trend in Europe and USA, if no new reactors come online and old ones operation licences are not renewed, nuclear power share in the electricity generation will fade away in the next decades (Tollefson, 2016).
Currently, expansion of nuclear power is being boost largely by emerging economies. Between 2011 and the end of 2016, thirty-seven out of the thirty-nine new operating reactors were based in China (23), Republic of South Korea (5), Russia (4), India (3), Pakistan (1), and Iran (1) (IAEA, 2017a). Nonetheless, experts flag up key concerns about China's dependence on nuclear infrastructure and technology about poor quality control, ineffective regulatory competence and limited construction experience (Thomas, 2017).

In this context, the World fleet of operating reactors is ageing. As for the end of 2017, the average age of operating reactors in the World was over 29 years. Further, sixty-four reactors (16\% of the total operating fleet) have been running for more than 40 years (IAEA, 2017a), and the extension of their lifetimes implies costly retrofits and envisages risks to their operational safety. It may also raise transboundary environmental issues and diplomatic tensions between neighbouring countries, as clearly observed in the plans of the Spanish government to grant a 10year extension to the Almaraz Nuclear Power Plant located only $100 \mathrm{~km}$ from the Portuguese border (World Nuclear Association, 2017).

Over the last decades, the nuclear sector has been promoting safer and more complex reactors (IAEA, 2017b; Morales Pedraza, 2017). However, none of the six different designs of generation IV reactors run

\footnotetext{
* Corresponding author at: Centre of Environmental Policy, Imperial College London, Weeks Building 16-18 Prince's Gardens, South Kensington, London SW7 1BA, UK.

E-mail addresses: j.portugal@imperial.ac.uk, joanaportugal@gmail.com (J. Portugal-Pereira).
} 
by the Generation IV International Forum (GIF) has entered in service and their investments are being revised or cancelled, which hinder their development. Further, most of the Generation III + reactors under construction, which include a set of advanced passive safety measures and more sophisticated engineering components, have been delayed. For instance, the Finish Olkiluoto 3 power reactor, a promising EPR technology, originally scheduled to be online in 2009, has suffered various delays. In the best-case scenario, it will start operating 10 years later (May 2019) than initially scheduled and with significant additional investments. In a similar line, in the UK, the Hinkley Point C power reactors, also an EPR technology, were planned to start operating in 2025 with initial construction cost of $20.3 \mathrm{bn}$ GBP. However, costs are estimated to be much higher as the government and EDF have already announced delays even before the start of the plant construction phase (NAO, 2017). Also, the small modular reactors (SMR), while a novel concept, still face considerable technical, economic and institutional challenges that will not be address in any near future time (Sovacool and Ramana, 2015).

Originally, the "learning-by-doing" concept was introduced by Arrow (1962) to explain productivity increases with experience for the same human capital and investment. Empirically, the literature identified several reasons for this effect, namely more labour efficiency, optimisation of design and supply chain and better use of technology/ equipment. This effect is generally observed in energy systems, such as in renewable energy technologies (strong evidences for solar-PV and wind-onshore). However, in the nuclear power sector, we witness a discontinuity of the learning curve. This is partly due to lack of standardisation, poor design and engineering planning, shortage of skilled labour, inadequate quality control, social concerns about nuclear risks, and shortage of private sector investments. All these factors result in highly uncertain projects of nuclear technology (Khatib and Difiglio, 2016; Sovacool et al., 2014a). Consequently, recent nuclear projects face high risks and a higher probability of longer lead-times. ${ }^{1}$ As for the end of 2016, there are fifty-four reactors under construction with an average lead-time of 8 years (IAEA, 2017a).

The hypothesis underneath this work is that, unlike other energy technologies, nuclear energy can actually lead to high overnight construction costs (OCC $)^{2}$ and longer lead-times given the uncertainty associated with tighten safety procedures and increasing complexity of last generation reactors. Over time, nuclear technology has become more complex, which required more labour and material intensive processes, raising construction and operation and maintenance (O\&M) costs. In addition, environmental licences and public acceptance are major reasons for construction delays and, therefore, overrun costs. This suggests a discontinuity of the learning curve effect in nuclear technology; i.e., accumulated experience results in a capital cost escalation rather than in a decline.

Several studies in the literature looked at learning curves for nuclear power and attempted to evaluate the effects of main cost drivers (Grubler, 2010; IEA, 2015; Jamasb, 2007; Kahouli, 2011; Koomey and Hultman, 2007; Kouvaritakis et al., 2000). However, learning curves are influenced by a vast range of factors and it is difficult to isolate specific learning effects (Khatib and Difiglio, 2016; Lovering et al., 2016).

Grubler (2010) and Berthélemy and Escobar Rangel (2015) provide economic analyses of nuclear reactor construction costs in France and in the United States based on overnight cost data. These studies conclude that, contrary to other energy technologies, innovation leads to

\footnotetext{
${ }^{1}$ Lead-time refers to time difference between the construction start time from the licensing procedures and the commercial operation time, when the reactor is connected to the grid after the initial test phase.

${ }^{2}$ OCC is defined as the construction costs as a project was implemented straightaway during day working hours and overnight. Therefore, this cost indicator is not sensitive to lead-time delays and consequently financial costs, financial structure of projects, interest rate during construction period and public subsidies.
}

increasing lead costs. In the same line, Sovacool et al. (2014b) investigate the frequency and magnitude of cost and time overruns occurring during the construction of electricity projects built over time, and concluded that nuclear reactors are the riskiest technology in terms of mean cost escalation as a percentage of budget and frequency. Cooper (2014) also evaluates nuclear power costs over time and concludes that most recent cost projections for new nuclear reactors are, on average, over four times as high as the initial nuclear reactors. In addition, Carvalho and Sauer (2009) compared the cost of nuclear power expansion in Brazil against other renewable and fossil fuel-based energy systems, concluding that nuclear is not the most cost-competitive option.

Lovering et al. (2016) also assess the experience curve of nuclear reactors. However, in their study the authors conclude that there is a positive effect learning curve of nuclear technology development. Similarly, IEA and NEA reports also show that nuclear power costs have dropped over time and will decline or remain flat in future (IEA, 2015; Varro and Ha, 2015). However, as highlighted in Koomey et al. (2017) and Gilbert et al. (2017), these studies assess the costs of reactors in an overnight basis, which do not reflect contingences and escalation of lead-time during construction in the overall construction costs. Therefore, overnight costs do not reflect the financial risks of investment costs or give a comprehensive picture of the cost trajectory over time. While relevant to the field, existing literature of nuclear power costs has mainly focused on reactors implemented in the USA and France, which account for one-third of the World operable reactors. Nonetheless, currently major construction of nuclear reactors occurs in emerging countries, mainly China, South Korea and India, which emphasises de need to expand previous analyses to emerging markets.

In sum, the state of the existing literature highlights the need for an analysis of the historical experience and trends of nuclear costs to assess the effect of time escalation, increasing safety measures and regulatory procedures as main proxies of escalation of costs in the nuclear technology.

In this context, this work seeks to analyse historical trends of leadtime, OCC and the cost of delay ( $\mathrm{CoD})$ of operable light water reactors (LWR). In addition, it aims to assess key factors that result in the discontinuity of the learning curve, including increasing complexity of technology, improved passive safety measures, tighten sectorial regulatory procedures, complex environmental licences, and public disapproval, especially after the Fukushima Dai-ichi accident. This analysis aims to show that the overnight cost of nuclear power projects is not a realistic indicator to assist decision makers and investors to incorporate the embedded risk and uncertainty underlined in large-scale projects.

Starting from a comprehensive characterisation of operable LWR reactor data set, this study updates and extends the OCC of Grubler (2010) and Lovering et al. (2016), assessing the learning effect on capital costs and lead-time through statistical analyses of pool data series. Furthermore, the CoD has been assessed through the additional financial costs that had to be incurred during the extended construction time from the moment of construction starts $\left(t_{0}\right)$ until the commercial operation $\left(t_{5+n}\right)$, considering contingences and unforeseen constrains.

The novelty of the study relies on the expansion of overnight construction costs (OCC) databases and the incorporation of financial cost analyses to assess the cost of delay of nuclear power plant (NPP) projects. As stated above, most of the available studies in the literature have focused on the analysis of overnight construction costs (OCC) of energy systems only. However, given the overrun time of energy mega projects, such as nuclear power plants, OCC is not an accurate proxy to evaluate the real costs of large-scale energy projects. To our best knowledge, this is the first attempt in the literature to conduct a systematic statistical treatment of overrun costs of construction of nuclear power reactors and to develop a comparative analysis of the cost of delay in different regions and periods of time. Furthermore, this study expanded the evaluation of NPP costs to the so-called "new 
racers", notably China, India, Pakistan, UAE and Iran.

This paper is organised as follows: following this brief overview of the current panorama of the World nuclear operable fleet, next section details the analytical framework adopted in this work, describing the developed characterisation database of operable, under construction and closed down reactors in the World and the statistical analysis conducted to estimate the lead-time, overnight construction costs, and the cost of delay. Section 3 shows the results and discusses the implications of results in the future of the World nuclear sector. This is followed by concluding remarks and policy recommendations.

\section{Analytical framework}

To assess the OCC, the lead-time and the CoD of the World nuclear reactor fleet, this work encompasses three stages, entailing: (i) design of a database of World nuclear reactor projects, (ii) statistical analysis of the OCC, lead-time and (iii) overrun costs of reactors over time.

\subsection{Database of existing nuclear power plants and planned future projects}

From the starting point of IAEA power reactor information system (PRIS) (IAEA, 2017a), an extensive list of the World nuclear reactor fleet has been collected. This encompasses 662 reactors, including phased-out, commercial operable, pilot, under construction and planned nuclear reactor projects from 1951 to 2016. The database covers not only key technological parameters, namely the net nominal capacity (GW), the capacity factor (\%) and reactor technology and model, lead-time (years), and operation starting year, but also other operational details, such as operator and supplier stakeholders. The OCC (US $\$_{2010} / \mathrm{kW} \mathrm{h}$ ) were collected, largely based on Lovering et al. (2016) and expanded based on IAEA reports (IAEA, 2016), WNA analyses (WNA, 2016) and country-based national reports. The present study expands past studies by including data from countries that are "new racers" in nuclear deployments, namely China, India, Pakistan, UAE and Iran. Data were normalised to US\$ in 2010 based on GDP deflators and official exchange rates from CIA World Factbook (CIA, 2016).

Reactors were then aggregated by technology type, model, operational status, country and decade of operation starting and compared at a global scale. This aims to assess relevant statistical parameters that dictate the trend of lead-time, OCC, and CoD from the early nuclear era of 1960 to present days.

Worldwide several nuclear reactor technologies have been developed, namely LWR, pressurised heavy water reactors (PHWR), gascooled reactors (GCR), and fast-breeder reactors (FBR). Over time, however, the nuclear sector has converged towards the LWR technology, which accounts for more than two-thirds of all operable reactors around the World (IAEA, 2017a). For this reason, among the overall nuclear reactors for which data were identified here, only LWR, i.e., pressurised water reactors (PWR) and boiling water reactors (BWR) units, have been assessed. Furthermore, the statistical analysis excludes pilot and demonstration reactors, as "first of a kind" reactors are not necessarily representative of commercial reactors. Also, reactors under construction, whose online operation is uncertain, have also been excluded because OCC reported do not reflect the effective cost of construction and are mere projections. This reduces the database to three hundred and eighty reactors, equivalent to $57 \%$ of the total World reactor fleet.

The geographical scope of the database includes the countries with the largest installed fleet among OECD countries, former Soviet countries, as well new comers, mainly emerging economies, including China, India, Republic of South Korea, UAE and Pakistan. Reactors were classified by technology generation according the construction year, including Generation I, II, and II + .

While data collection and compilation was an objective and comprehensive process, readers should be aware that this study relies mainly on sources from governments, sectorial institutions, project promotors and implementers. Given that the nuclear power sector is poorly overseen, it may be the case that initial OCC projections do not necessarily translate reliable data. However, to the authors' best knowledge, there is no comprehensive and unbiased database of construction costs associated with nuclear power reactors worldwide. While the IAEA publishes data on aggregated technical and operational parameters of the World nuclear fleet, it does not detail construction cost data. Lovering et al. (2016) put a great effort to compile cost data, but the authors also strongly rely on data from governmental sources and nuclear agencies. However, the eventual "noise" introduced by OCC data inputs has limited impacts on the results of this study, as the assessment of the financial costs (Section 2.3) is based on overrun leadtime, which is precisely reported by the IAEA (2017a).

As for the end of 2016, thirty-one countries operate nuclear power plants or have plants under construction. Three hundred and sixty-eight LWR reactors are currently operable with $343 \mathrm{GW}$ of installed capacity, which include the long-term outage reactors of the Japanese fleet and the Swedish Oskarshamn-2, whose restart is uncertain. Further, fiftyone new reactors are under construction and planned to start up until 2021 , totalising an added installed capacity of $54 \mathrm{GW}$. Since the beginning of the nuclear era, fifty-nine reactors have been shut downed, mainly in Germany (19), in the USA (15), and Japan (8) (IAEA, 2017a). The consolidated database is included in the supplementary materials of this paper.

\subsection{Statistical analysis of overnight construction costs and lead-time data series}

Two indicators (OCC and lead-time) have been subject of a statistical analysis. The software package @RISK 7.0 from Palisade (2015) has been applied to run the statistical tests of the data series to identify the probability distribution function that best fits the data. The model that best fits to explain the statistical distribution of overnight construction costs and construction time is not known a priori. For this reason, the Akaike information criterion (AIC) (Akaike, 1973) was used for that purpose. When selecting a model that best fits among several possible models, it must be borne in mind that there are no true models. There are only approximate models that cause loss of information. Thus, it is necessary to make the selection of the "best" model, among those that were adjusted, to explain the phenomenon under study. The AIC criterion helps then to make this selection in a more scientific way, since it compares the quality of a set of statistical models to each other and choses the model that best fits to the data set.

\subsection{Evaluation of overrun costs}

In addition to the OCC indicator, the overrun cost has also been estimated. Unlike the OCC, the overrun cost includes the interest and financial charges during the delayed construction period besides the intrinsic cost of the technology per se. This is an essential indicator to reflect the realistic costs of mega projects, such as nuclear power plants, given their susceptibility to unexpected delays and high capital risks (Callegari et al., 2018). Although past studies and the nuclear industry typically exclude this indicator (see Lovering et al., 2016; Grubler, 2010), the overrun cost from lead-time delays is a fundamental factor to explain the escalation of nuclear technology costs. As noted by Gilbert et al. (2017), Koomey et al. (2017), and Sovacool et al. (2014b), the cost of delay contributes significantly for overrun costs in the implementation of nuclear reactor projects due to overseen contingences. Missing to assess the implications of regulatory, political, design and management contingences and associated delays in the reactor construction is therefore a crude simplification that does not accurately reflects the full costs of nuclear projects.

Furthermore, the differences observed between OCC and CoD may also be explained considering a complexity of factors commonly defined 
in the literature as the "planning fallacy", as firstly proposed by Kahneman and Tversky (1979), or the "Malevolent Hiding Hand", as identified by Flyvbjerg and Sunstein (2015). Both principles explain that predictions of future projects tend to underestimate their time needed to be completed and underline an optimism bias based on certain heuristics. Planners tend to underestimate the time, costs, and risks of future projects, which results in unexpectedly high costs and benefit shortfalls. In this line, Kahneman and Lovallo (1993) also suggest that many failures of projects originate in bold forecasts, which undervalue past risk analyses and negative exposures.

Actually, the literature reveals that large-scale technical and social complex projects can face higher risks of cost overruns and construction delays (de Bruijn and Leijten, 2008). Overrun costs are intensified by "the strategy of making too low an estimate of the costs involved in the mega project in order for the decision-making to go more smoothly at this stage" (de Bruijn and Leijten, 2008), which seems to be the case for projects justified by their strategic importance" (Flyvbjerg et al., 2013).

Therefore, the final investment decision (FID) of many nuclear projects, seen as "strategic" in emerging and developed economies (Fam et al., 2014; Thomas, 2017), is based on inaccurate cost estimates and even on the lack of construction experience with modern reactor designs (Thomas, 2017). In sum, cost estimation, inaccuracies of FID, technological complexities and lack of social acceptability (de Bruijn and Leijten, 2008; de Groot et al., 2013) are all part of a panoply of reasons behind OCC in the nuclear industry history.

To estimate the CoD a discounted cash flow analysis has been developed, considering four major factors: (i) projected OCC, (ii) the leadtime of each nuclear reactor, (iii) the weighted distribution of capital cost during the construction period, and (iv) the average discount rate for power generation projects. The projected OCC and lead-time of nuclear projects have been complied in an extensive database, as described in Section 2.1. The weighted distribution of investment costs follows a sinusoidal function (Fig. 1), as proposed by MIT (2003). This sinusoidal distribution has been originally applied to a standard leadtime of five years and then translocated through a horizontal vector $(\Delta t$, 0 ) to reflect the time of delay assuming a common distribution of the OCC. The discount rate utilised is one of the major influences in the overall costs of energy projects, especially in highly intensive capital projects, such as nuclear plants. In this study, an average discount rate of 10\% per year was assumed, following Khatib and Difiglio (2016).

\section{Results and discussion}

\subsection{Lead-time and overnight construction costs}

Figs. 2 and 3 and illustrate the evolution of the lead-time and OCC

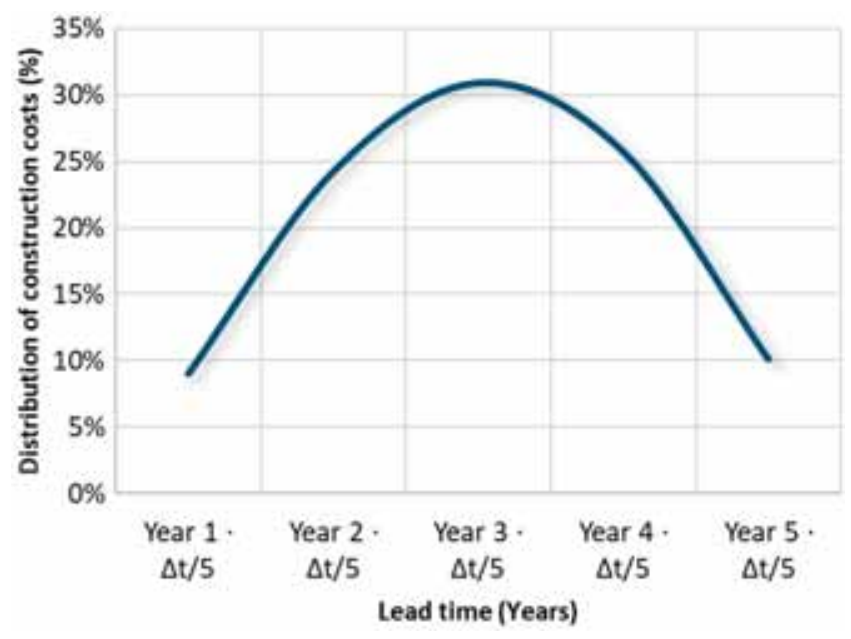

Fig. 1. Profile of capital cost distribution of nuclear reactors.
(US\$2010 $/ \mathrm{kW}$ ), respectively, of selected PWR and BWR units from the early nuclear era to the end of 2016. Both figures present a trend line for indicative purposes, showing a positive slope over time for both OCC and lead-time. Results reveal a general increasing trend of OCC and lead-time from the 1970s to the 2010s. While OCC and lead-time were fairly regular during the 1970s and 1980s, recent nuclear projects OCC and construction time vary significantly. Although data are very disperse and this trend may be influenced by extreme high values, the minimum values tend to follow a similar trend, suggesting a discontinuity of the learning curve. In addition, the lead-time and OCC dispersion trend increases over time, which reinforces the hypothesis of higher uncertainty and risks of nuclear investments. These results go against the analysis of Lovering et al. (2016), but are supported by other views in the literature (Gilbert et al., 2017; Grubler, 2010; Koomey and Hultman, 2007; Sovacool et al., 2014a). A detailed analysis of drivers behind the problems associated to individual nuclear projects is beyond the scope of this study. Nonetheless, a general assessment by Schneider and Froggatt (2017) concludes that complex licensing procedures in most countries, financing negotiations, site preparation and other delays in the infrastructure development are major factors behind nuclear power reactor construction delays. In addition, as stated above, implementers tend to underestimate the OCC due to technical, economic, psychological, and political factors.

Figs. 4 and 5 reveal in detail the lead-time and OCC, respectively, of PWR and BWR disaggregated by decades. While in the 1960s, a nuclear reactor would be constructed in average in less than five years (4.6), with an associated OCC of 1723 US $\$ 2010 / \mathrm{kW}$, in the 2000 s the mean of lead-time climbed up to an average of more than ten years (10.6), with a mean OCC of 2637 US $\$_{2010} / \mathrm{kW}$. This suggests a twofold increase in lead-time in the last 40 years. More recently, between 2011 and 2016, the lead-time declined to levels below 8 years. While lower than the previous period in the decade of 2000s, it still reveals an escalation of lead-time when compared to the 1960s.

There is also an escalation of the square deviation from 1.3 years in the 60 s to 7.5 years between 2011 and 2016 and the increasing number of maximum outliners, which suggests a higher deviation in the leadtimes and riskier investments for nuclear power projects. The rise of lead-times of PWR and BWR is clearly reflected in the OCC. In the early 60 s and 70s, the OCC were averagely $60 \%$ of the one observed between 2011 and 2016. This goes in line with the hypothesis that, over time, nuclear projects have become more expensive due to an increasing complexity of technology, more efficient control mechanisms and more safety measures. The increasing needs of safety systems and more complex nuclear power reactors also raises the labour and material intensity of the construction process. Although there is not a World nuclear reactor material database or a detailed life cycle assessment available to quantify, with precision, the materials required per MW generated for different nuclear power reactor technologies, empirical assessments of Gen III + and IV reactors suggest that those more complex and advanced than early stage reactors.

This discontinuity in the learning curve has different implications across regions (Figs. 6 and 7). Regional factors that may influence the OCC and lead-time include different national regulatory systems, local institutions, public acceptability of nuclear projects and specific location factors, such as availability of cooling water, seismic activity, labour and material costs (Khatib and Difiglio, 2016).

In more centralised-planned and vertically integrated power systems, such as in China and Japan, the lead-time is shorter than in other regions. This is mainly due to a strong centralised government, public investment and fast licencing process, given the limited public participation in the process and poor regulation of the nuclear safety system (Sun et al., 2016; Wu, 2017). As highlighted by Zhou and Zhang (2010) public in China has a general positive support of nuclear energy, as a strategy to reduce air pollution in urban centres. Currently, the majority of new nuclear reactors under construction are in China, which seems to keep a good record of on-time construction period. 


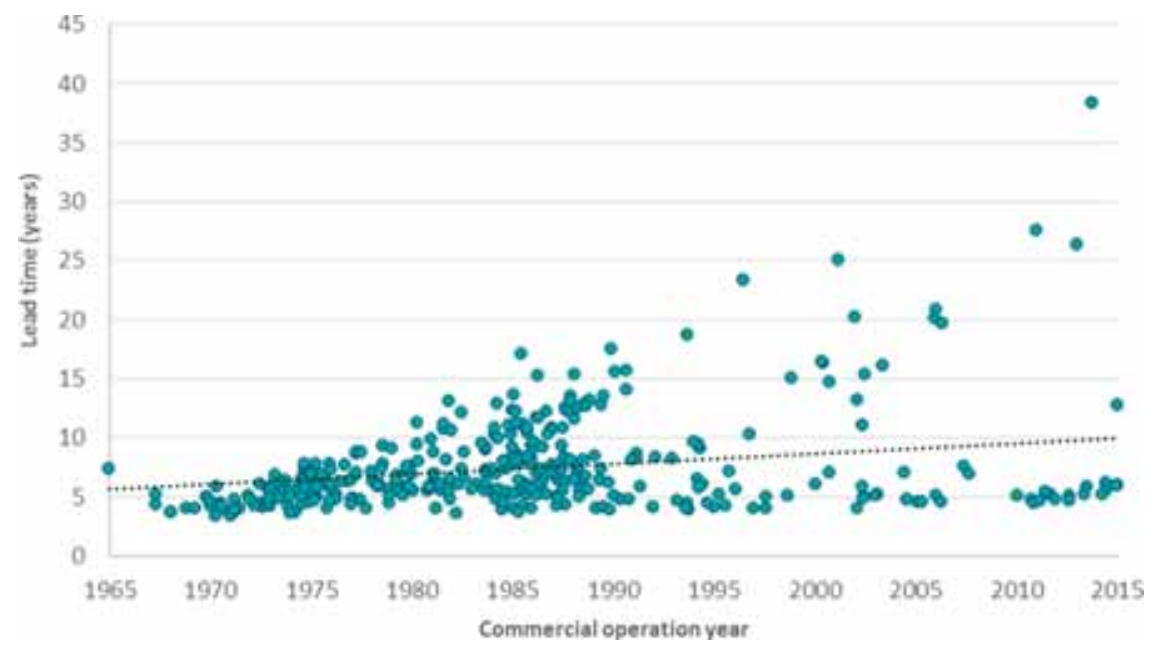

Fig. 2. Lead-time of PWR and BWR units (years).

Japan's reactor fleet shows a similar trend, with a fairly stable leadtime, nonetheless with evidence of OCC escalation among the analysed years. Yet, it is important to highlight that the evaluated data series (until 2016) does not reflect the on-hold nuclear projects, idle reactors and retrofitting costs in the aftermath of the Fukushima Dai-ichi accident. For instance, the Hamaoka nuclear power plant, operated by Chubu Electric Power Co, was requested to implement more resistance tsunami breakwater walls at a cost of JPY 400 billion (US $\$ 3.7$ billion) (Esteban and Portugal-Pereira, 2014; NRA, 2013; World Nuclear Association, n.d.). Furthermore, the 12 planned new nuclear reactors, totalising $4.1 \mathrm{GW}$, are unlike to be operating in the future, given the sceptical public opinion regarding nuclear safety in Japan and the willness to expand the installed capacity of wind and solar technologies (Esteban et al., 2018; Portugal Pereira et al., 2014).

In Western European countries, such as France, the standardisation of the nuclear fleet and nuclear projects simplified the licencing processes and the implementation of the projects. In general, the West European reactor fleet follows a standardised design, which accelerates the regulatory assessments and licencing process. Nonetheless, Western European countries also reveal an increase of costs and high number of outliners. For instance, France has done extensive efforts of standardisation of its 58 reactors in order to simplify the licence process and reduce construction costs. Yet, as Grubler (2010) demonstrated, real costs ramped up over the last decades.

On the other hand, the costs of nuclear projects in the USA reveal one of the highest costs and remarkably pronounced long lead-time. In

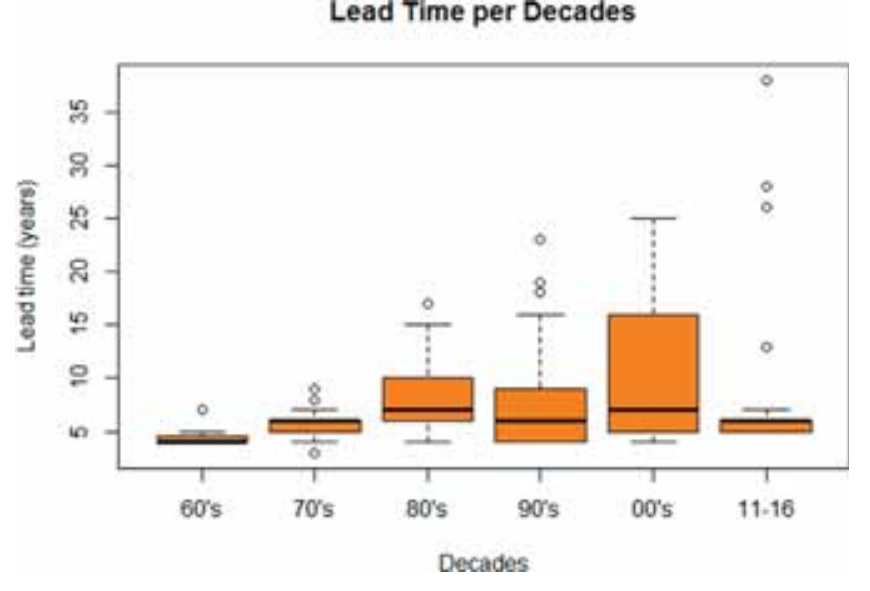

Fig. 4. Lead-time of PWR and BWR units per decade.

fact, in the USA there is a large dispersion in the standardisation of reactor design. Therefore, the benefits of "learning by doing" are not evident. Eastern European regions, which include a large number of exsoviet countries and Russia, reveal high lead-time and OCC and a great number of outliers, mainly due to the political instability in the region and certain number of on-hold projects.

OCC and lead-time indicators seem to follow a similar distribution,

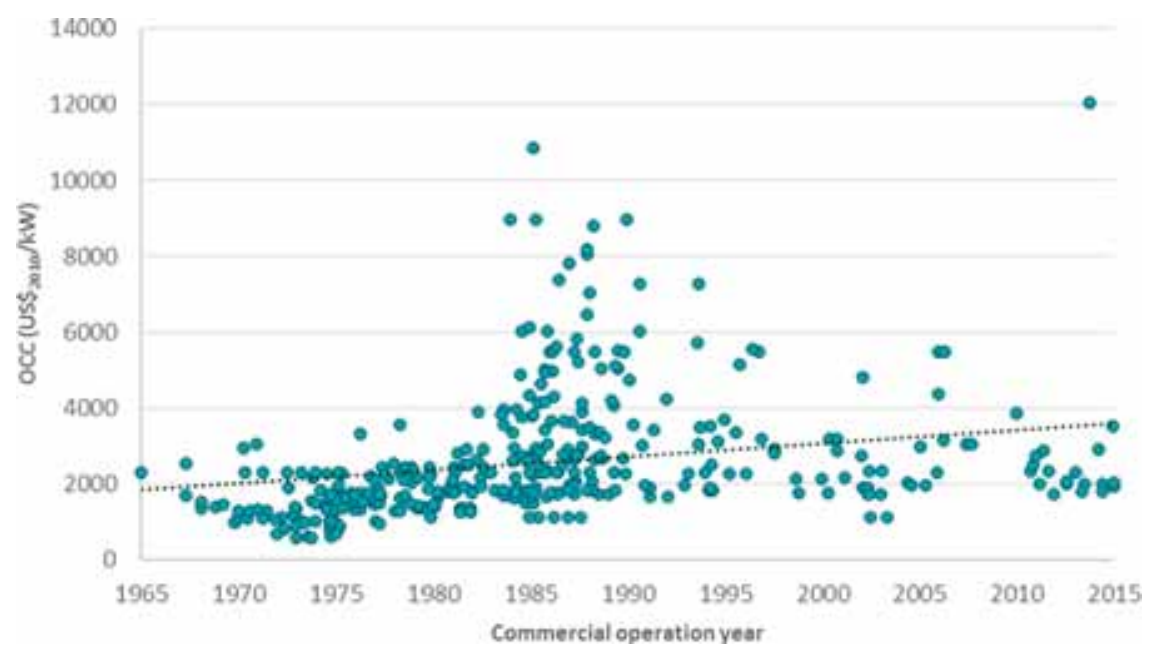

Fig. 3. OCC of PWR and BWR units (US $\$_{2010} / \mathrm{kW}$ ). 


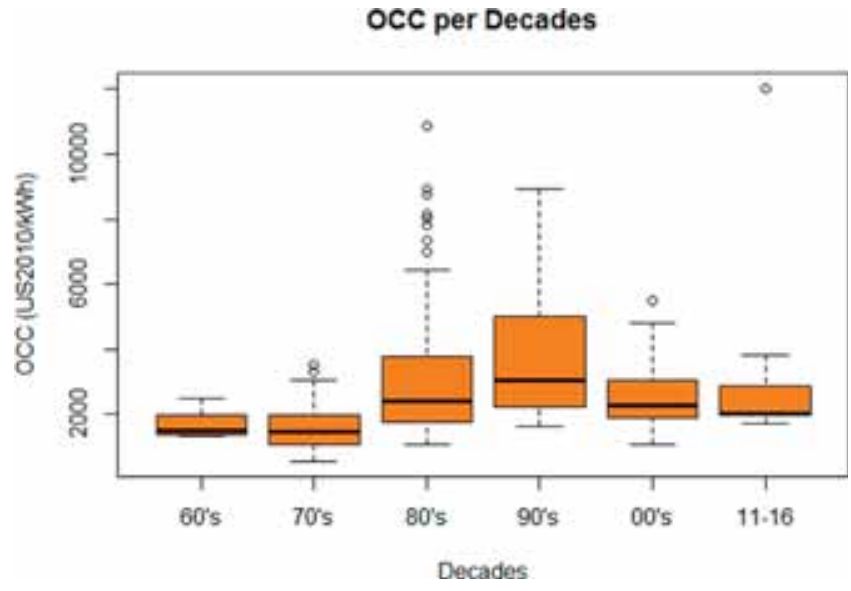

Fig. 5. OCC of PWR and BWR units per decade (US $\$ 2010 / \mathrm{kW})$.

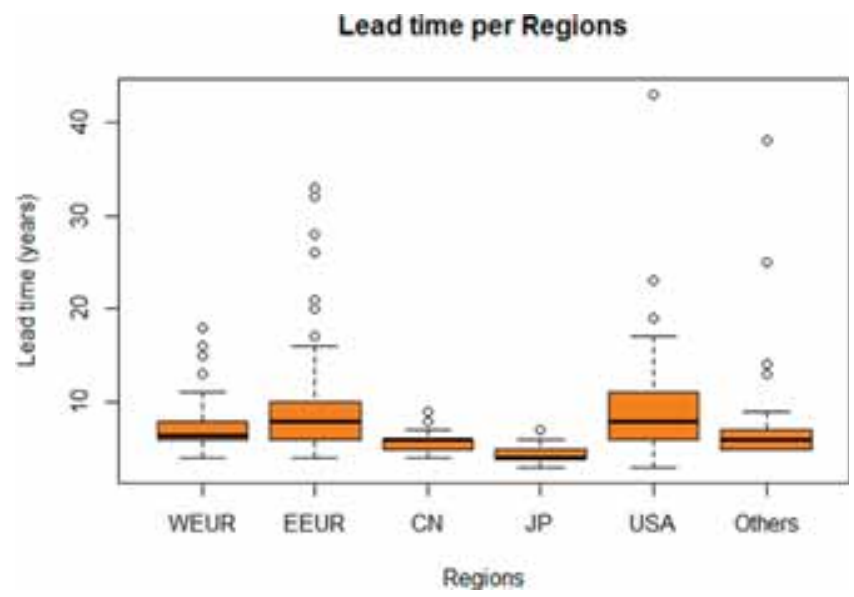

Fig. 6. Lead-time of PWR and BWR units per region (years). WEUR - Western Europe; EEUR - Eastern Europe; CN - China; JP - Japan; USA - United States of America.

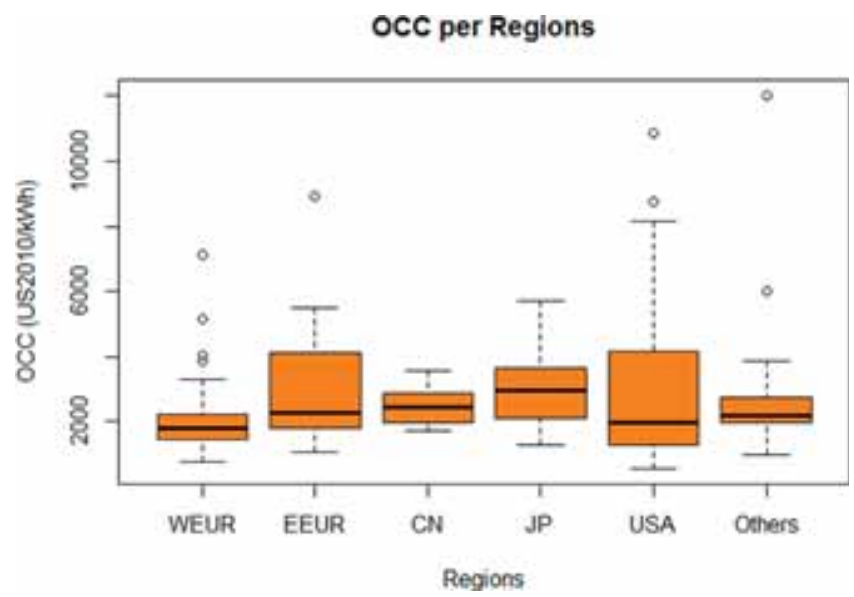

Fig. 7. OCC of PWR and BWR units per region (US $\$_{2010} / \mathrm{kW}$ ). WEUR - Western Europe; EEUR - Eastern Europe; CN - China; JP - Japan; USA - United States of America.

giving rise to the hypothesis that these parameters may be related. The correlation coefficient between OCC and lead-time for data series is 0.48 , which may be considered a value on the border of "weak" to "significant" correlation (Suomalainen et al., 2015). It can be justified by the increasing complexity of the nuclear technology resulting in escalation of lead-time, higher OCC, possible overruns and additional financing costs.

An interesting outcome is the positive significant correlation (0.29) between lead-time and the reactor size, corroborating Berthélemy and Escobar Rangel (2015) results. The average construction time for small reactors $(<900 \mathrm{MW})$ is 5.9 years, with a standard deviation of 2.5. Large reactors ( $>900 \mathrm{MW}$ ), on the other hand, present a lead-time of 8.2 years, and a standard deviation of 4.4 , which translates into higher uncertainty and consequently higher OCC. This goes in line with previous finding in the literature (Callegari et al., 2018; Sovacool et al., 2014b), reinforcing that the possible economies of scale of larger reactors are eroded by costs overruns and construction delays.

As for the OCC, the correlation with reactor size is positive but weak (0.22), which shows that relying on the OCC for the evaluation of large reactors can be a too optimistic approach. The importance of financial costs incurred during the construction time is a relevant factor not to be neglected on a realistic planning and evaluation exercise.

Figs. 8 and 9 corroborate the similar shape of probability distribution function (pdf) of OCC and construction time series as both of them can be adjusted to lognormal functions. Although a high concentration of values can be found around the average, the positive long tail on the right shows a high dispersion of the database and the probability of reaching a construction time or an OCC higher than the corresponding average is more than $35 \%$. This distribution shape represents energy projects with highly risk parameters, such as nuclear reactors.

\subsection{The cost of delay}

For the calculation of the cost of delay (CoD), the profile of capital cost of nuclear reactors was kept unchanged and assumed as a normal distribution (Fig. 10). The financial cost of delay was computed against the standard lead-time of five years for a discount rate of $10 \%$ per year, departing from the calculation of the total cost including financial costs incurred during the construction time (TOCC) and the total cost including financial costs for a given standard construction time (SOCC). Eqs. (1)-(3) summarise the proposed model for the economic valuation of the delay.

SOCC $=\sum_{\mathrm{t}=1}^{\mathrm{ST}} \mathrm{OCC}_{\mathrm{t}}(1+\mathrm{i})^{(\mathrm{ST}-t)}$

$\mathrm{TOCC}=\sum_{\mathrm{t}=1}^{\mathrm{LT}} \mathrm{OCC}_{\mathrm{t}}(1+\mathrm{i})^{(\mathrm{LT}-t)}$

$\mathrm{CoD}=\mathrm{TOCC}-\mathrm{SOCC}$

where $S T$ represents standard construction time (assumed as five years in this analysis), $O C C_{t}$ stands for the value of OCC expected to be spent on year $t$ and $L T$ represents the lead-time of the reactor.

The results indicate that more than $76 \%$ of the analysed projects present a delay comparatively to the standard lead-time and that the value of the cost of this delay represents on average more than $18 \%$ of the TOCC. Fig. 10 describes the probability distribution function of the total cost and shows that the adjustment against a lognormal function of TOCC is increasingly evident. Compared to Figs. 8 and 10 shows a longer right tail, reflecting higher investment risks embedded in the financial costs of nuclear power projects. This demonstrates the importance of assigning a monetary value to the delay. An important fact to retain is the high value of the standard deviation, reflecting the high impact of both OCC and lead-time factors taken together to assess the overrun risk of nuclear power projects.

The economic impact of a construction cost overrun is the possible loss of the economic justification for the project. A cost overrun can also be critical to policies for pricing electricity based on economic costs, because such overruns would lead to under-pricing. The financial impact of a cost overrun is the strain on the power utility and on national financing capacity in terms of foreign borrowings and domestic credit. For this reason, this analysis shows that nuclear power projects involve 


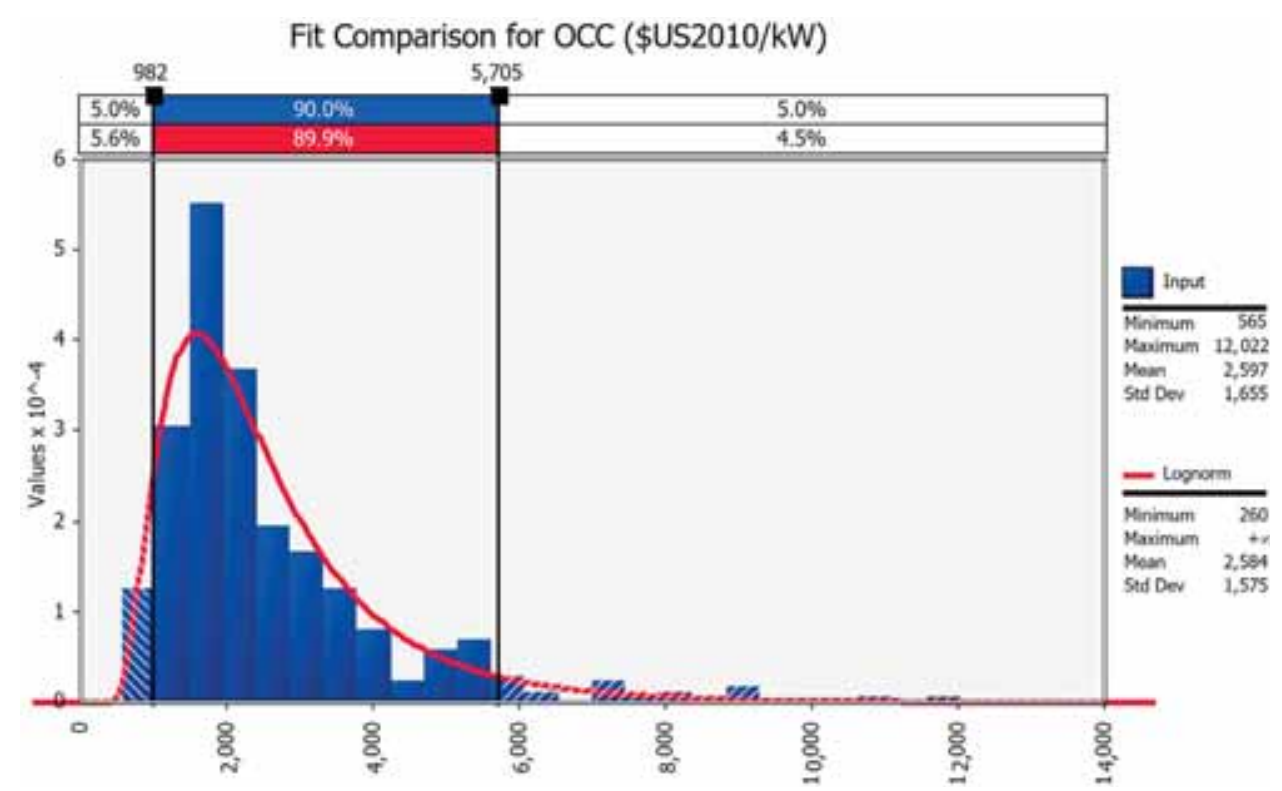

Fig. 8. Probability distribution function (pdf) for OCC of PWR and BWR nuclear reactors (\$US 2010).

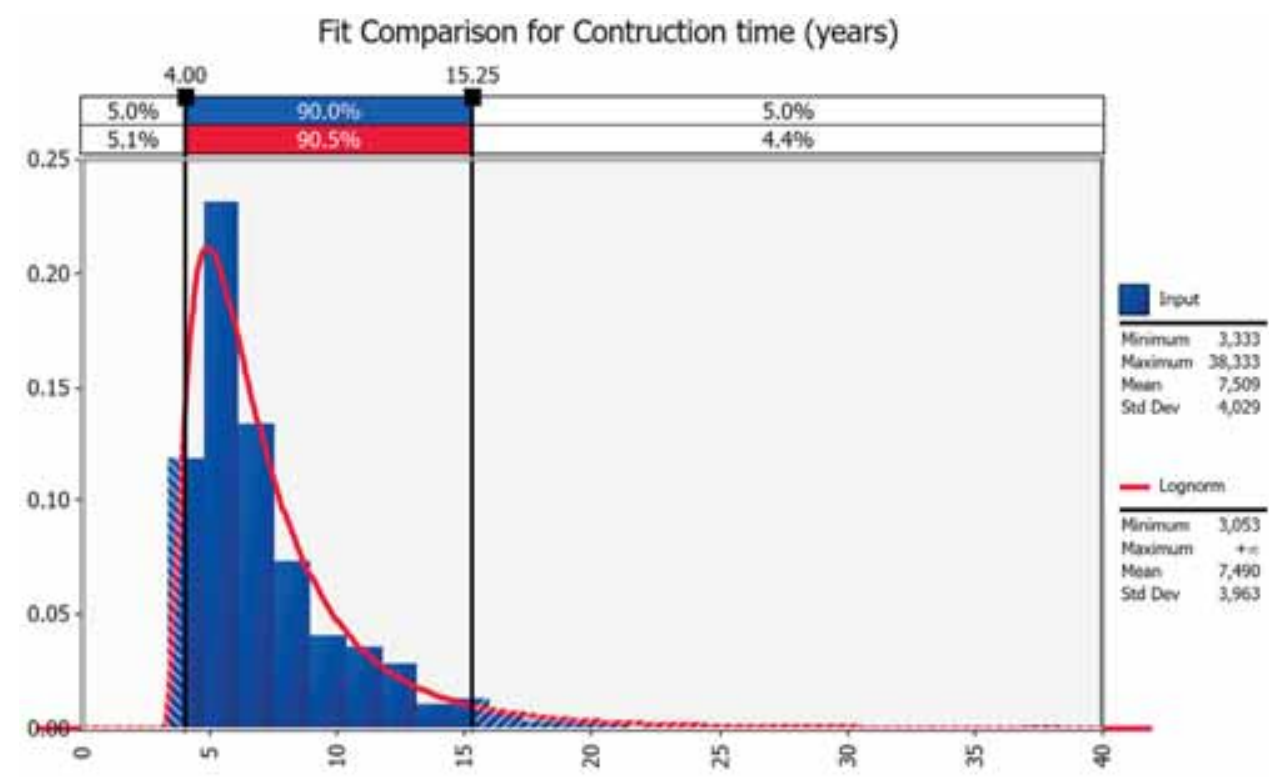

Fig. 9. Probability distribution function (pdf) for construction time of PWR and BWR nuclear reactors (years).

unforeseen risks that result in complex and highly uncertain energy investments. While such a fate does not seem to be specific to nuclear power plants, but to energy mega projects in general (Callegari et al., 2018), nuclear power plants still stand as the emblematic case of failed energy mega projects.

The complexity of energy mega projects may come from the close interaction of a wide range of drivers, including technical, environmental, governmental, technological and social factors, that make it difficult to manage the different interfaces to reach the initial goals (Remington and Zolin, 2011). Complexity may also be explained as the interdependence between several factors that are interrelated and have multiple objectives and actors (Olaniran et al., 2015). It occurs when technical, environmental, and legal factors, among others, interact so closely that managing their interactions becomes difficult. Thence, the level of complexity of a project grows as the number of interrelationships and interdependencies among the different factors increases.

\section{Concluding remarks and policy recommendations}

The accumulated experience with nuclear reactor technology does not necessarily translate into a learning curve. Over time, there is a trend of more complex reactors with safer passive systems and tighten regulatory procedures. The increasing complexity and uncertainty of nuclear projects suggest higher OCC and longer lead-time. This work sought to evaluate the effects of these parameters in the OCC, lead-time and the cost of delay of nuclear reactors from 1955 to 2016. To this end, a comprehensive database of commercial LWR reactors was developed and a statistical analysis was conducted using the @Risk project risk management software. To our best knowledge, this is the first attempt in the literature to conduct a systematic statistical treatment of overrun costs of construction of nuclear power reactors and to develop a comparative analysis of the cost of delay in different regions and periods of time.

There are significant delays in lead-time, which increases over time, especially for the last generation reactors constructed in the 2010s. 


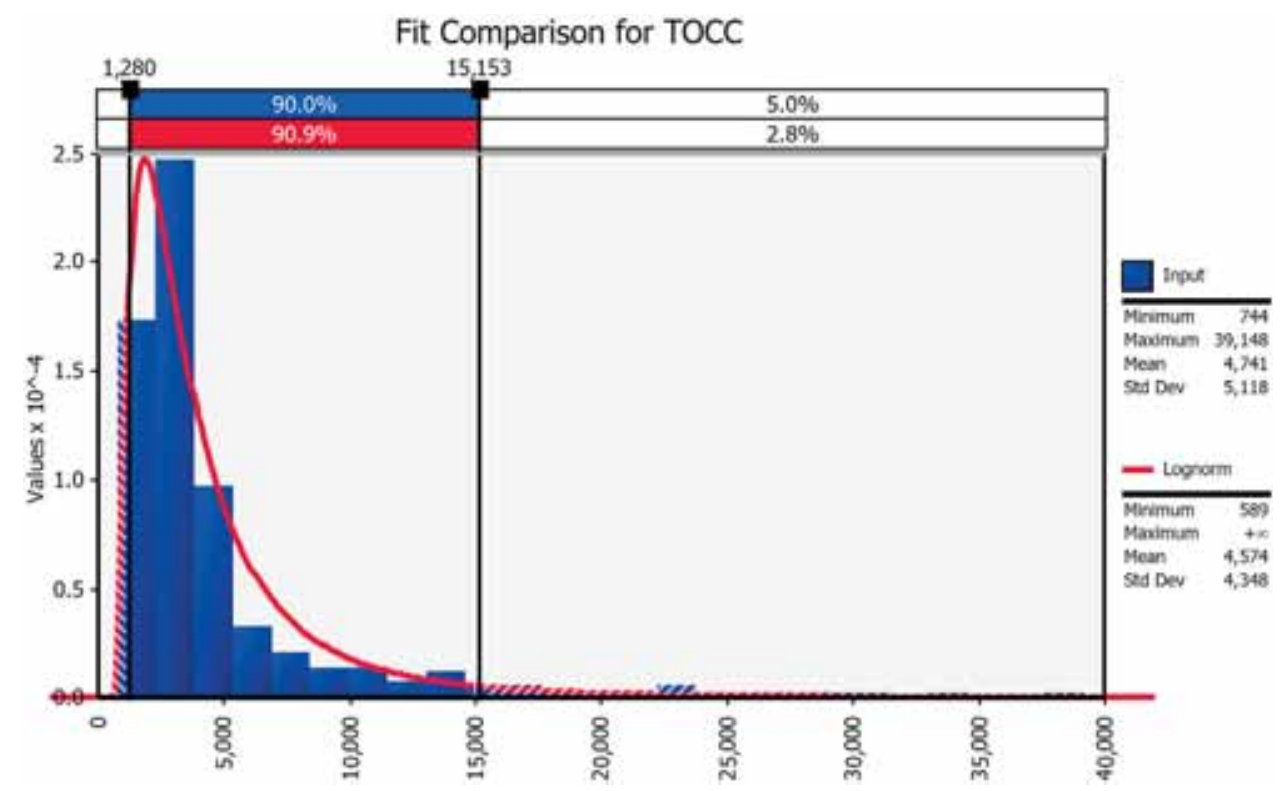

Fig. 10. Probability distribution function (pdf) of OCC including financial costs incurred during the construction time (\$US 2010).

Results showed that $3 / 4$ of the analysed projects present a delay as compared to the standard lead-time. This leads to escalation of OCC rather than a decline. Average OCC of newer reactors are considerably higher than the ones implemented in the earlier stages of the nuclear era. This finding suggests that the nuclear technology is significantly costly and takes too long to be implemented, which increases the project risks, given its high probability of cost overrun and construction delays. This threats the market and the financial sustainability of future and current nuclear energy projects. Therefore, nuclear technology is not at the forefront to cope with the need for climate change mitigation strategies to contribute to decarbonising of the power sector. Unlike IAEA ambitious forecasts on the role of nuclear power in the global energy mix to achieve the Paris Agreement goals (IAEA, 2017), nuclear power underlines high investment risks and uncertainty.

Given the lead-time delays and escalation of OCC, this study suggests that the analysis of financial costs is an effective strategy to evaluate meaningful direct construction costs of nuclear reactors. Among the analysed nuclear construction projects, the cost of delay, assessed as the financial costs, represents on average more than $18 \%$ of the TOCC. This is a clear message to decision makers and private investors to rethink their energy investment strategies and shift capital flows towards more secure, and sustainable, low-carbon projects.

Energy forecasts suggest that the electricity consumption will continue to increase steadily over time given a growing global economy and given an irreversible trend towards the electrification of energy end use sector. Therefore, it is true that, when it comes to building new power projects, it is better late than never. Nevertheless, the matter of truth is that never late is even better, particularly to address the climate change mitigation challenge. Policymakers, project developers, implementers and nuclear sector agencies should rethink hand in hand the future implementation of nuclear power plant projects. This should include: (i) adoption of better planning tools to avoid optimist bias and over simplification of project implementation steps, (ii) development of more transparent regulation processes to guarantee the safe and reliable operation of nuclear reactors, (iii) improvement of current nuclear construction cost databases with more reliable and trustful data, and (iv) expansion of current databases with site-specific data, such as life cycle material requirements of the construction of new reactors and labour intensity during all stages of the reactor life cycle, including the decommission stage.

Despite our efforts to conduct an accurate analysis of overrun costs of the World's nuclear reactor fleet, we recognise that our study has limitations that can be dealt with in future works, to enhance the robustness of our findings, namely:

- Analysis of the so-called hidden costs or indirect costs, which includes investments on R\&D and community level compensations to host nuclear power reactor projects. In countries such as Japan and South Korea, for example, implementers invest on community facilities, such as hospitals, cultural centres, among others, as a compensation to local population directly affected by nuclear power reactors construction and operation risks;

- A discount rate of $10 \%$ per year was assumed in the financial analysis, but this is an oversimplification, as different regions may apply different discount rates depending upon site-specific contexts;

- The weighted distribution of investment costs was considered a sinusoidal function and translocated uniformly through the overrun construction period. Nonetheless this is a theoretical simplification, since it is not possible to acquire site-specific data on overrun leadtime and construction status; and,

- OCC and financial cost analysis followed a top-down approach relying on institutional, governmental and sectorial data. Adopting a bottom-up methodology, such as life cycle material and cost assessments, would evaluate site-specific data, which could refine results and detailed socioeconomic drivers of NPP construction delays.

\section{Acknowledgments}

This work was funded by the Brazilian National Council for Scientific and Technological Development agency (CNPq) (166074/ 2015-2) and the Marie Curie International Research Staff Exchange Scheme Fellowship within the $7^{\text {th }}$ European Union Framework Programme, under the project NETEP- European Brazilian Network on Energy Planning (PIRSES-GA-2013-612263). Authors would like to acknowledge Rafael Garaffa for his helpful advices about processing data with $\mathrm{R}$ statistical software and data analysis. Authors are also thankful to the comments of anonymous reviewers. Special thanks to the Grantham Institute and Imperial College London media office for assisting in publicising the findings of the paper. 


\section{Appendix A. Supporting information}

Supplementary data associated with this article can be found in the online version at http://dx.doi.org/10.1016/j.enpol.2018.05.041.

\section{References}

Akaike, H., 1973. Information theory and an extension of the maximum likelihood principle., in: Petrov, B.N., Caski, F. (Eds.), Proceedings of the Second International Symposium on Information Theory Pp. 267-281. Akademiai Kiado, Budapest, pp. 267-281.

Aoki, M., Rothwell, G., 2013. A comparative institutional analysis of the Fukushima nuclear disaster: lessons and policy implications. Energy Policy 53, 240-247. http:// dx.doi.org/10.1016/j.enpol.2012.10.058

Arrow, K., 1962. The Economic Implications of Learning by Doing. Rev. Econ. Stud. 29, 155-173.

Berthélemy, M., Escobar Rangel, L., 2015. Nuclear reactors' construction costs: the role of lead-time, standardization and technological progress. Energy Policy 82, 118-130. http://dx.doi.org/10.1016/j.enpol.2015.03.015.

Callegari, C., Szklo, A., Schaeffer, R., 2018. Cost overruns and delays in energy mega projects: how big is big enough? Energy Policy 114, 211-220. http://dx.doi. org $/ 10.1016 /$ j.enpol.2017.11.059.

Carvalho, J.F., Sauer, I.L., 2009. Does Brazil need new nuclear power plants? Energy Policy 37, 1580-1584. http://dx.doi.org/10.1016/j.enpol.2008.12.020.

CIA, 2016. The World Factbook [WWW Document]. Cent. Intell. Agency.

Cooper, M., 2014. The Economic Failure of Nuclear Power and the Development of a Low Carbon Electricity Future: Why Small Modular Reactors are Part of the Problem Not the Solution.

de Bruijn, H., Leijten, M., 2008. Management Characteristics of Mega-Projects. In: Priemus, Hugo, Flyvbjerg, Bent, van Wee, Bert (Eds.), Decision-Making on MegaProjects. Edward Elgar Publishing.

de Groot, J.I.M., Steg, L., Poortinga, W., 2013. Values, perceived risks and benefits, and acceptability of nuclear energy. Risk Anal. 33, 307-317. http://dx.doi.org/10.1111/ j.1539-6924.2012.01845.x.

Esteban, M., Portugal-Pereira, J., 2014. Post-disaster resilience of a $100 \%$ renewable energy system in Japan. Energy 68, 756-764. http://dx.doi.org/10.1016/j.energy. 2014.02.045.

Esteban, M., Portugal-Pereira, J., Mclellan, B.C., Bricker, J., Farzaneh, H., Djalilova, N., Ishihara, K.N., Takagi, H., Roeber, V., 2018. 100\% renewable energy system in Japan: Smoothening and ancillary services. Appl. Energy 224, 698-707. http://dx.doi.org/ 10.1016/j.apenergy.2018.04.067. (ISSN 0306-2619). https://www.sciencedirect. com/science/article/pii/S0306261918306299.

Fam, S.D., Xiong, J., Xiong, G., Yong, D.L., Ng, D., 2014. Post-Fukushima Japan: the continuing nuclear controversy. Energy Policy 68, 199-205. http://dx.doi.org/10. 1016/J.ENPOL.2014.01.014.

Flyvbjerg, B., Holm, M.K.S., Buhl, S.L., 2013. Underestimating Costs in Public Works Projects: Error or Lie? 〈http://dx.doi.org/10.1080/01944360208976273〉.

Flyvbjerg, B., Sunstein, C.R., 2015. The Principle of the Malevolent Hiding Hand; or, the Planning Fallacy Writ Large.

Gilbert, A., Sovacool, B.K., Johnstone, P., Stirling, A., 2017. Cost overruns and financial risk in the construction of nuclear power reactors: a critical appraisal. Energy Policy 102, 644-649. http://dx.doi.org/10.1016/j.enpol.2016.04.001.

Grubler, A., 2010. The costs of the French nuclear scale-up: a case of negative learning by doing. Energy Policy 38, 5174-5188. http://dx.doi.org/10.1016/j.enpol.2010.05. 003 .

Huenteler, J., Schmidt, T.S., Kanie, N., 2012. Japan's post-Fukushima challenge - implications from the German experience on renewable energy policy. Energy Policy 45, 6-11. http://dx.doi.org/10.1016/j.enpol.2012.02.041.

IAEA, 2017a. Power reactor information system (PRIS): The Database on Nuclear Power Reactors [WWW Document].

IAEA, 2017b. International Status and Prospects for Nuclear Power 2017. GOV/INF/ 2017/12-GC(61)/INF/8.

IAEA, 2017c. Energy, Electricity and Nuclear Power Estimates for the Period up to 2050. Reference data series No. 1. IAEA-RDS-1/37. Internacional Atomic Energy Agency (IAEA), Vienna, Austria.

IAEA, 2016. Nuclear Power Reactors in the World. IAEA-RDS-2/36. Viena, Austria. doi:ISBN 978-92-0-103716-9.

IEA, 2015. Technology Roadmap: Nuclear Energy. Paris, France.

Jamasb, T., 2007. Technical change theory and learning curves: patterns of progress in electricity GenerationTechnologies. Energy J. 28, 51-72.

Kahneman, D., Lovallo, D., 1993. Timid choices and bold forecasts: a cognitive perspective on risk taking. Manag. Sci. 39, 17-31. http://dx.doi.org/10.1287/mnsc.39. 1.17 .

Kahneman, D., Tversky, A., 1979. Prospect theory: an analysis of decision under risk. Econometrica 2, 263-291.

Kahouli, S., 2011. Effects of technological learning and uranium price on nuclear cost: preliminary insights from a multiple factors learning curve and uranium market modeling. Energy Econ. 33, 840-852. http://dx.doi.org/10.1016/j.eneco.2011.02. 016.

Khatib, H., Difiglio, C., 2016. Economics of nuclear and renewables. Energy Policy 96, 740-750. http://dx.doi.org/10.1016/j.enpol.2016.04.013.

Koomey, J., Hultman, N.E., 2007. A reactor-level analysis of busbar costs for US nuclear plants, 1970-2005. Energy Policy 35, 5630-5642. http://dx.doi.org/10.1016/j. enpol.2007.06.005.

Koomey, J., Hultman, N.E., Grubler, A., 2017. A reply to "Historical construction costs of global nuclear power reactors.”. Energy Policy 102, 640-643. http://dx.doi.org/10. 1016/j.enpol.2016.03.052.

Kouvaritakis, N., Soria, A., Isoard, S., 2000. Modelling energy technology dynamics: methodology for adaptive expectations models with learning by doing and learning by searching. Int. J. Glob. Energy Issues 14, 104-115.

Lovering, J.R., Yip, A., Nordhaus, T., 2016. Historical construction costs of global nuclear power reactors. Energy Policy 91, 371-382. http://dx.doi.org/10.1016/j.enpol.2016. 01.011.

MIT, 2003. The future of nuclear power: an interdisciplinary MIT study.

Morales Pedraza, J., 2017. Advanced Nuclear Technologies and Its Future Possibilities. In: Morales Pedraza, Jorge (Ed.), Small Modular Reactors for Electricity Generation: An Economic and Technologically Sound Alternative. Springer International Publishing, Cham, pp. 35-122. http://dx.doi.org/10.1007/978-3-319-52216-6_2.

NAO, 2017. Hinkley Point C. HC 40 Session 23/06/2017. London. UK.

NRA, 2013. Enforcement of the New Regulatory Requirements for Commercial Nuclear Power Reactors.

Olaniran, O.J., Love, P.E.D., Edwards, D., Olatunji, O.A., Matthews, J., 2015. Cost overruns in hydrocarbon megaprojects: a critical review and implications for research. Proj. Manag. J. 46, 126-138. http://dx.doi.org/10.1002/pmj.21556.

Palisade, 2015. @Risk assessment software [WWW Document].

Portugal-Pereira, J., Troncoso Parady, G., Castro Dominguez, B.,, 2014. Japan's energy conundrum: Post-Fukushima scenarios from a life cycle perspective. Energy Policy 67, 104-115. http://dx.doi.org/10.1016/j.enpol.2013.06.131.

Remington, K., Zolin, R., 2011. Controlling chaos? The value and the challenges of applying complexity theory to project management. In: Cooke-Davies, T. (Ed.), Aspects of Complexity: Managing Projects in a Complex World. Project Management Institute, Newtown Square, Pa.

Schneider, M., Froggatt, A., 2017. The World Nuclear Industry - Status Report 2017. Paris, France.

Soda, S., Hamada, M., Kuno, M., 2017. Future Technology for the Seismic Safety of Nuclear Power Facilities, in: Earthquake Engineering for Nuclear Facilities. pp. 229-245. 〈http://dx.doi.org/10.1007/978-981-10-2516-7_12〉.

Sovacool, B.K., Gilbert, A., Nugent, D., 2014a. An international comparative assessment of construction cost overruns for electricity infrastructure. Energy Res. Soc. Sci. 3, 152-160. http://dx.doi.org/10.1016/j.erss.2014.07.016.

Sovacool, B.K., Gilbert, A., Nugent, D., 2014b. Risk, innovation, electricity infrastructure and construction cost overruns: testing six hypotheses. Energy 74, 906-917. http:// dx.doi.org/10.1016/j.energy.2014.07.070.

Sovacool, B.K., Ramana, M.V., 2015. Back to the future: small modular reactors, nuclear fantasies, and symbolic convergence. Sci. Technol. Hum. Values 40, 96-125. http:// dx.doi.org/10.1177/0162243914542350.

Srinivasan, T.N., Rethinaraj, T.S.G., 2013. Fukushima and thereafter: reassessment of risks of nuclear power. Energy Policy 52, 726-736. http://dx.doi.org/10.1016/j. enpol.2012.10.036.

Sun, C., Zhu, X., Meng, X., 2016. Post-Fukushima public acceptance on resuming the nuclear power program in China. Renew. Sustain. Energy Rev. 62, 685-694. http:// dx.doi.org/10.1016/j.rser.2016.05.041.

Suomalainen, K., Pritchard, G., Sharp, B., Yuan, Z., Zakeri, G., 2015. Correlation analysis on wind and hydro resources with electricity demand and prices in New Zealand. Appl. Energy 137, 445-462. http://dx.doi.org/10.1016/j.apenergy.2014.10.015.

Thomas, S., 2017. China's nuclear export drive: Trojan Horse or Marshall Plan? Energy Policy 101, 683-691. http://dx.doi.org/10.1016/j.enpol.2016.09.038.

Tollefson, J., 2016. Nuclear power plants prepare for old age. Nature 537, 16-17. http:// dx.doi.org/10.1038/537016a.

Varro, L., Ha, J., 2015. Projected Costs of Generating Electricity - 2015 Edition. Paris, France.

Vivoda, V., 2012. Japan's energy security predicament post-Fukushima. Energy Policy 46, 135-143. http://dx.doi.org/10.1016/j.enpol.2012.03.044.

WNA, 2016. World nuclear association - facts and figures [WWW Document].

World Nuclear Association, 2017. Nuclear Power in Spain [WWW Document]. Ctry. profiles.

World Nuclear Association, n.d. Nuclear Power in Japan [WWW Document]. 2016.

Wu, Y., 2017. Public acceptance of constructing coastal/inland nuclear power plants in post-Fukushima China. Energy Policy 101, 484-491. http://dx.doi.org/10.1016/j. enpol.2016.11.008.

Zhou, S., Zhang, X., 2010. Nuclear energy development in China: a study of opportunities and challenges. Energy 35, 4282-4288. http://dx.doi.org/10.1016/j.energy.2009.04. 020 . 